\title{
Effect of Small Focus on Electron Heating and Proton Acceleration in Ultrarelativistic Laser-Solid Interactions
}

\author{
N. P. Dover® ${ }^{1,{ }^{*}}$ M. Nishiuchi, ${ }^{1,2}$ H. Sakaki, ${ }^{1}$ Ko. Kondo, ${ }^{1}$ M. A. Alkhimova,${ }^{3}$ A. Ya. Faenov,${ }^{4,3}$ M. Hata, ${ }^{5}$ \\ N. Iwata, ${ }^{5}$ H. Kiriyama, ${ }^{1}$ J. K. Koga $\odot,{ }^{1}$ T. Miyahara, ${ }^{1,6}$ T. A. Pikuz ${ }^{4,3}$ A. S. Pirozhkov $\odot,{ }^{1}$ A. Sagisaka, ${ }^{1}$ \\ Y. Sentoku, ${ }^{5}$ Y. Watanabe, ${ }^{6}$ M. Kando $\odot,{ }^{1}$ and K. Kondo ${ }^{1}$ \\ ${ }^{1}$ Kansai Photon Science Institute, National Institutes for Quantum and Radiological Science and Technology, \\ Kizugawa, Kyoto 619-0215, Japan \\ ${ }^{2}$ PRESTO, Japan Science and Technology Agency, 4-1-8 Honcho, Kawaguchi, Saitama 332-0012, Japan \\ ${ }^{3}$ Joint Institute for High Temperatures, Russian Academy of Sciences, Moscow 125412, Russia \\ ${ }^{4}$ Open and Transdisciplinary Research Initiative, Osaka University, Suita, Osaka 565-0871, Japan \\ ${ }^{5}$ Institute of Laser Engineering, Osaka University, 2-6 Yamadaoka, Suita, Osaka 565-0871, Japan \\ ${ }^{6}$ Interdisciplinary Graduate School of Engineering Sciences, Kyushu University, Kasuga, Fukuoka 816-8580, Japan
}

(Received 25 January 2019; revised manuscript received 17 December 2019; accepted 18 December 2019; published 26 February 2020)

\begin{abstract}
Acceleration of particles from the interaction of ultraintense laser pulses up to $5 \times 10^{21} \mathrm{~W} \mathrm{~cm}^{-2}$ with thin foils is investigated experimentally. The electron beam parameters varied with decreasing spot size, not just laser intensity, resulting in reduced temperatures and divergence. In particular, the temperature saturated due to insufficient acceleration length in the tightly focused spot. These dependencies affected the sheath-accelerated protons, which showed poorer spot-size scaling than widely used scaling laws. It is therefore shown that maximizing laser intensity by using very small foci has reducing returns for some applications.
\end{abstract}

DOI: 10.1103/PhysRevLett.124.084802

The interaction of high intensity laser pulses with solid targets is fundamental to many applications of high power lasers including energetic ion and x-ray sources [1-3], fast ignition inertial confinement fusion [4-6], radiobiology $[7,8]$, materials science $[9,10]$, and others [11,12]. Progress in high power laser technology [13] has recently enabled experimental investigation at intensities approaching the radiation-reaction dominated and QED regime [14], and facilities are coming online to demonstrate applications based on such interactions.

When ultraintense lasers (normalized vector potential $a_{0} \gg 1$ ) interact with solids, electrons are accelerated to relativistic energies by the extreme laser fields $[15,16]$. Estimating the electron energy is complicated by field patterns generated by the focusing and reflected laser [17], plasma fields [18,19], and prepulse induced preplasma $[20,21]$. A commonly used scaling law to predict the increase in electron temperature, $T_{e}$, with laser intensity $I_{L}$ is the ponderomotive scaling $T_{\text {pond }}=\left(\gamma_{t}-1\right) m_{e} c^{2}$, where $\gamma_{t}=\sqrt{\left(1+a_{0}^{2}\right)}$, the maximum transverse kinetic energy in

Published by the American Physical Society under the terms of the Creative Commons Attribution 4.0 International license. Further distribution of this work must maintain attribution to the author(s) and the published article's title, journal citation, and DOI. a plane wave [15]. However, other theories based on energy conservation [22], time-averaged momentum [16], and empirical scalings [23] predict worse scaling. The scaling of this and other electron parameters to ultrahigh intensities is not clear, particularly in the $\sim \mathrm{fs}$ pulse regime.

One key application of electron heating is target normal sheath acceleration (TNSA), or sheath acceleration, of ions [24-27]. Although other acceleration mechanisms have been investigated such as radiation pressure acceleration [28,29], collisionless shockwave acceleration [30-32], and relativistically induced transparency or breakout afterburner [33-35], sheath acceleration is attractive for applications due to its simplicity, robustness, and smooth beam profile. Electrons propagate through a thin foil, generating a quasielectrostatic field on the target surface which accelerates ions. Various acceleration models predict ion energies based on electron flux, temperature, and divergence [36-45]. To understand model efficacy, experimental studies on laser-electron coupling and ion acceleration at the high intensity frontier are crucial for realizing the potential of next generation high power lasers.

In this Letter, we investigate the laser intensity scaling of electron and proton acceleration in laser-solid interaction up to $I_{L} \approx 5 \times 10^{21} \mathrm{~W} \mathrm{~cm}^{-2}\left(a_{0} \approx 50\right)$. We demonstrate that electron temperature depends not only on laser intensity, but also on focal spot size, which restricts the electron acceleration length and therefore temperature. 
We find reduced electron divergence for tightly focused laser pulses due to beam generated magnetic fields in the preplasma. We demonstrate that the resultant sheath accelerated proton energies increase only modestly with decreased focal size due to reducing acceleration time. Increasing laser energy, not just laser intensity, may be the only route for achieving significant increase to maximum proton energies without employing more sophisticated acceleration schemes.

An $f / 1.4$ off-axis parabolic mirror focused the $0.1 \mathrm{~Hz}$, $\tau_{L} \approx 40 \mathrm{fs}, E_{L} \approx 10 \mathrm{~J}$ (on target), $\lambda_{L} \approx 800 \mathrm{~nm}$ J-KAREN$\mathrm{P}$ laser [46-48] to a focal size $r_{L} \approx 1.5 \mu \mathrm{m}$ (FWHM), resulting in a peak $I_{L}=5 \times 10^{21} \mathrm{~W} \mathrm{~cm}^{-2}$, measured at full power [46]. A motorized tape provided a $d=5 \mu \mathrm{m}$ thick stainless steel foil at $45^{\circ}$ to the $p$-polarized laser. The intensity was controlled by reducing $E_{L}$ or moving the target with respect to the laser focus, measuring $I_{L}$ at the offset. This setup allowed accumulation of a relatively large dataset compared to that typically used in ultrahigh intensity laser-solid interaction experiments ( $\sim 100$ shots).

The experiment is shown in Fig. 1(a) [49]. The electron spectrum is measured along the laser axis using a magnetic spectrometer with a phosphor scintillator (Mitsubishi DRZ), and the electron beam spatial profiler used another scintillator filtered by $6 \mathrm{~mm}$ polytetrafluoroethylene, eliminating ions, and $<2 \mathrm{MeV}$ electrons. The proton spectrum was measured along target normal with a time-of-flight (TOF) diagnostic with $5 \mathrm{~m}$ drift length [56], with a scintillatorbased proton beam profile monitor ensuring target normal pointing [59]. Radiochromic film (RCF) stacks could be inserted for high dynamic range spatially resolved spectroscopy [60], confirming the TOF measurement.

The laser prepulse causes pre-expansion of the target, affecting the main pulse interaction. The laser contrast was measured using a third order cross-correlator, and its effect on the target was calculated using FLASH [49]. The axial density profile $10 \mathrm{ps}$ before the main pulse is shown in Fig. 1(b) for different laser conditions, showing a characteristic double scale length transitioning near the critical density $n_{c} \approx 10^{21} \mathrm{~cm}^{-3}$ where the prepulse is strongly absorbed. Because of transverse heat transport, a small variation of laser parameters does not significantly affect the

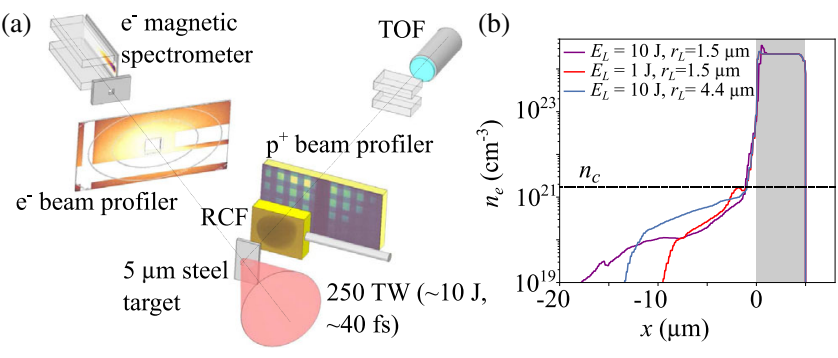

FIG. 1. (a) Experimental setup and (b) simulated prepulsedriven target expansion in different conditions 10 ps before main pulse. preplasma. In the preplasma, the proportion of contaminant protons to ions is less than a few percent, and therefore front side acceleration is dominated by the more abundant target ions, resulting in inefficient acceleration [58].

Typical electron spectra are shown in Fig. 2(a) for three shots: $I_{L} \approx 5 \times 10^{21} \mathrm{~W} \mathrm{~cm}^{-2}$, and reduced intensity $I_{L} \approx$ $6 \times 10^{20} \mathrm{~W} \mathrm{~cm}^{-2}$ by defocusing $\left(r_{L} \approx 5 \mu \mathrm{m}\right)$, or reducing energy $\left(r_{L} \approx 1.5 \mu \mathrm{m}\right)$. At the highest intensity, the high energy tail has a temperature $T_{e} \approx 12 \mathrm{MeV}$ [least square fitting of $d N / d E \propto \exp \left(E / T_{e}\right)$ at $\left.E>7 \mathrm{MeV}\right]$, significantly lower than $T_{\text {pond }} \approx a_{0} m_{e} c \approx 24 \mathrm{MeV}$ predicted from the ponderomotive theory. When $I_{L}$ is reduced by increasing $r_{L}$ at fixed energy, the $T_{e} \approx 9 \mathrm{MeV}$ is close to the ponderomotive scaling prediction $\approx 8 \mathrm{MeV}$. Unexpectedly, for the same $I_{L}$ but a smaller $r_{L}, T_{e}$ is reduced to $\approx 5 \mathrm{MeV}$. The temperature not only depends on $I_{L}$, but also $r_{L}$. Data from a single shot sequence in which the energy and focal spot were varied are shown in Fig. 2(b). Although $T_{e}$ follows ponderomotive scaling at low intensities, it is suppressed as the intensity increases. Furthermore, suppression is more significant for the small $r_{L}$ used when varying $E_{L}$.

A typical electron beam spatial profile is shown in Fig. 2(c) at highest intensity. The electrons are directed near laser axis with divergence $\theta_{e} \approx 30^{\circ}$ (FWHM), similar
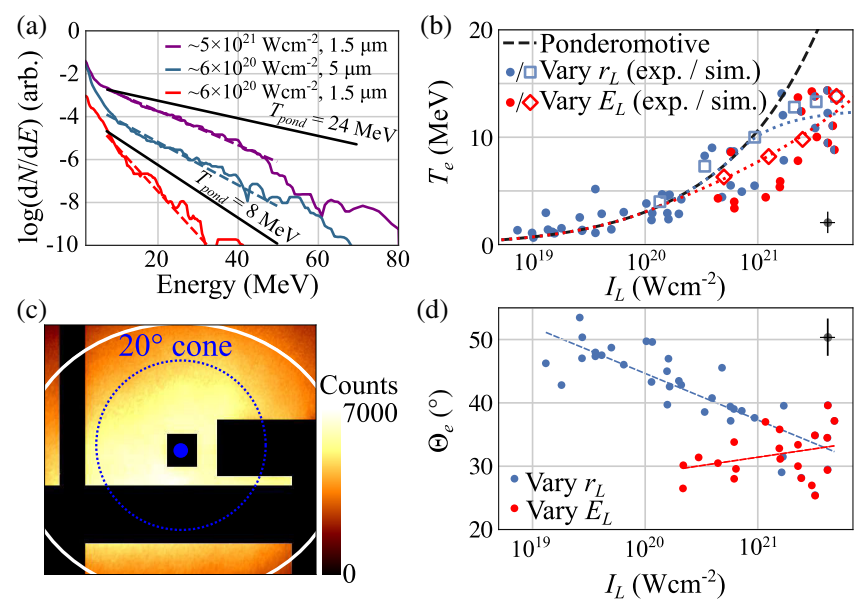

FIG. 2. (a) Electron spectrum for best focus full energy (purple), larger focus full energy (blue), best focus reduced energy (red), $T_{e}$ fits (dashed), and ponderomotive prediction $T_{\text {pond }}$ (black). (b) Experimental measurements of $T_{e}$ against $I_{L}$ by changing $r_{L}$ (blue circles), or $E_{L}$ at best focus (red circles), typical uncertainty (black cross) and respective PIC results (empty blue square, red diamond). The black dashed line is ponderomotive scaling, and the blue and red dotted lines the modified scaling. (c) Typical electron spatial profile (best focus and full energy). The blue dot and ring are laser axis and angular scale, white ring is the fitted Gaussian FWHM. Black regions were blocked by other diagnostics. (d) Experimental measurements of $\theta_{e}$ against $I_{L}$ for changing $r_{L}$ (blue circles) or $E_{L}$ (red circles), typical experimental uncertainty (black cross) and linear fits to guide the eye (dashed). 
to or smaller than observations for longer pulse lasers at similar energies [64-66]. As shown in Fig. 2(d), lowering $I_{L}$ by increasing $r_{L}$ results in increased electron divergence, contrasting with previous measurements at lower intensities and larger focal spots [67]. However, using a tight focus and varying $I_{L}$ by changing $E_{L}$ showed little variation, indicating a process inherent to the laser geometry. Therefore, we have shown a spot size dependence on both electron temperature and divergence.

The impact of $r_{L}$ has not previously appeared in electron heating models. For an electron in a plane wave, the characteristic transverse and longitudinal acceleration length, $y_{0} \propto a_{0}$ and $x_{0} \propto a_{0}^{2}$, can exceed the focal size bounded by $r_{L} \sim \lambda_{L}$ and Rayleigh length $x_{R} \sim \pi \lambda_{L}$ typical of high intensity lasers. Electron energy gain, given by the work done by the transverse electric field $W_{y}=$ $-\int_{c}|e| E_{y} d y$, can therefore be suppressed due to insufficient acceleration length.

However, actual field distributions are complicated by focusing, reflection, and plasma effects. It has been shown that longitudinal oscillating electric fields $[68,69]$ or static plasma magnetic [70] and electric fields [71,72] affect electron heating. Therefore, particle-in-cell (PIC) simulations were performed using EPOCH2D [73]. A box of $60 \times 60 \mu \mathrm{m}$ was simulated with spatial resolution $10 \times 20 \mathrm{~nm}$. A $\tau_{L}=40$ fs laser was focused on the target with varied $r_{L}$ and $I_{L}$ using p polarization unless otherwise specified. The target was $d=5 \mu \mathrm{m}$ preionized iron at $45^{\circ}$ angle of incidence, with 50(4) particles per cell for electrons(ions). To minimize numerical heating, the peak target electron density was restricted to $250 n_{c}$, still significantly higher than $n_{\mathrm{cr}}$. To isolate the electron acceleration physics, the same preplasma conditions were used for all simulations described below [Fig. 1(b) blue line]. Additional simulations using the preplasma profiles from Fig. 1(b) showed variation in $T_{e}$ of $<10 \%$.

The focal region is shown in Fig. 3(a) for best focus conditions $\left(r_{L}=1.5 \mu \mathrm{m}, I_{L}=5 \times 10^{21} \mathrm{~W} \mathrm{~cm}^{-2}\right)$. The laser penetrates the preplasma and is reflected at the relativistic critical density $n_{e} \approx n_{\mathrm{cr}}$, with no appreciable self-focusing. The electrons within the focal spot are driven along the laser axis and generate a strong azimuthal magnetic field $\bar{B}_{z}>40 \mathrm{kT}$ [74]. This pinching field is even more evident in simulations with the laser polarization rotated out-ofplane (s-pol.). As evidenced by the overlaid electron trajectories, forward accelerated electrons are reflected by this field towards the laser axis [75], reducing their divergence $[18,76]$. The electron distribution is exponential above $>5 \mathrm{MeV}$, and fitting a temperature at the pulse peak including all simulation particles, gives $T_{e} \approx 11 \mathrm{MeV}$, significantly less than $T_{\text {pond }} \approx 24 \mathrm{MeV}$ and matching the experimental observation.

Increasing $r_{L}$ with fixed intensity causes $T_{e}$ to increase until it reaches the ponderomotive scaling [Fig. 3(b), blue circles]. Despite the same field strength, using a small focus
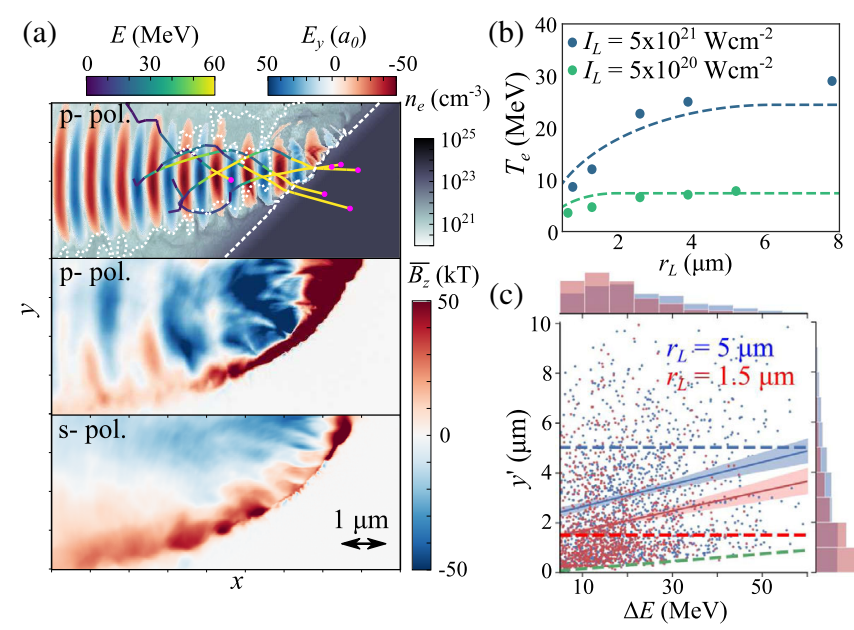

FIG. 3. From PIC: (a) $n_{e}$ and $E_{y}$ (visible where $a_{0}>10$ ) at the pulse peak, with dotted and dashed lines representing $n_{c}$ and $n_{\text {cr }}=a_{0} n_{c}$, respectively, and representative electron traces overlaid (top). Laser-cycle averaged magnetic field $\overline{B_{z}}$ for simulations with $\mathrm{p}$ and s polarization (middle and bottom). (b) $T_{e}$ against $r_{L}$ for two values of $I_{L}$, and respective model prediction [dotted line, Eq. (1)]. (c) $y^{\prime}$ against $\Delta E$ for $I_{L}=6 \times 10^{20} \mathrm{~W} \mathrm{~cm}^{-2}$ and $r_{L}=1.5,5 \mu \mathrm{m}$ of 1300 traced electrons $>5 \mathrm{MeV}$ (red, blue respectively). The dashed lines are the respective focal spot sizes, solid lines are a linear regression fit, and the green dashed line is $y_{\min }^{\prime}$.

has affected not just the number, but also the average kinetic energy of the electrons. Suppression of $T_{e}$ at small focal spots is also apparent at lower intensities $\left[5 \times 10^{20} \mathrm{~W} \mathrm{~cm}^{-2}\right.$ Fig. 3(b), green circles], agreeing with the model [Eq. (1)]. Further simulations varying focal spot size and energy over the experimental range are shown in Fig. 2(b), reproducing the trend. As the focal spot increases, the azimuthal magnetic field which reduced the electron divergence at small $r_{L}$ [Fig. 3(b)] weakens due to decreasing current density and increase in focal size to much larger than the preplasma spatial scale. The resultant electron momentum distribution extends to larger angles, also seen experimentally.

Following individual electron trajectories shows that the complex field pattern at the front surface results in rapidly varying accelerating trajectories, with most electrons experiencing semistochastic accelerating phases before entering the target [77]. On average, the majority of work was done by the transverse field [ $>80 \%-90 \%$, depending on focusing geometry (see Supplemental Material [49] for more detail), depending on focusing geometry], therefore electrons require a transverse acceleration length $y^{\prime}=\int\left|v_{y}\right| d t$ to gain energy. In Fig. 3(c), $y^{\prime}$ during acceleration (defined as when $5 m_{e} c^{2}<E<E_{\max }$ ), is plotted against electron energy gain $\Delta E$ for two different focal sizes for $I_{L}=6 \times 10^{20} \mathrm{~W} \mathrm{~cm}^{-2}$, showing strong positive correlation. The most energetic electrons are accelerated over a path significantly larger than the minimum 
$y_{\min }^{\prime}=\Delta E / e E_{L}$ (green dashes). As indicated by the histograms, the smaller focal spot leads to lower acceleration lengths, despite the self-generated azimuthal field, and therefore lower energies.

Although describing average electron dynamics in such a complex field pattern is analytically challenging, a heuristic model based on ponderomotive scaling can replicate the observed $T_{e}$. For a plane wave, an electron has a maximum transverse momentum $p_{y}=a_{0} m_{e} c$ and transverse acceleration distance $y_{0}=a_{0} \lambda_{L} / 2 \pi$. In the ponderomotive model [15], the electron temperature is set to the transverse kinetic energy of the electron, $T_{e}=$ $\left[\sqrt{1+\left(p_{y} / m_{e} c\right)^{2}}-1\right] m_{e} c^{2}$. Instead, if acceleration ceases at $y=r_{L e}$, where $r_{L e}=r_{L} /(2 \sqrt{\ln 2})$ is the radius where the intensity reduces by $1 / e$, then by rewriting $p_{y}$ in terms of $y$ and setting $p_{y}=y=0$ before acceleration, the final momentum at $y=r_{L e}$ is

$$
p_{f}=a_{0} m_{e} c\left[1-\left(1-\frac{r_{L e}}{y_{0}}\right)^{2}\right]^{1 / 2}
$$

for $r_{L e}<y_{0}$. When $r_{L e}>y_{0}$, there is no restriction by the field size and $p_{f}=a_{0} m_{e} c$; however, for $r_{L e} \ll y_{0}$ the momentum saturates. Using $p_{y}=p_{f}$ for calculating $T_{e}$ predicts the saturation of $T_{e}$ and the $r_{L}$ dependence seen experimentally [dotted lines Fig. 2(b)] and numerically [dashes Fig. 3(b)].

An important application of electron heating in lasersolid interactions is ion acceleration in the surface sheath, which depends on the electron source parameters discussed above. The maximum proton energy $E_{p}$ against $E_{L}$ $\left(r_{L} \approx 1.5 \mu \mathrm{m}\right)$ and $r_{L}\left(E_{L} \approx 10 \mathrm{~J}\right)$ is plotted in Figs. 4(a) and 4(b). Increasing $E_{L}$ shows an approximate power law scaling $E_{p} \propto E_{L}^{0.6} \propto I_{L}^{0.6}$, weaker than observed previously at lower laser energy for $\sim \mathrm{fs}$ lasers [43]. However, increasing intensity by decreasing $r_{L}$ shows even weaker scaling, $E_{p} \propto r_{L}^{-1 / 2} \propto I_{L}^{1 / 4}$. Focusing to $r_{L}<5 \mu \mathrm{m}$ has neither increased $T_{e}$, nor significantly boosted the maximum proton energy $E_{p}$ despite increasing $I_{L}$.
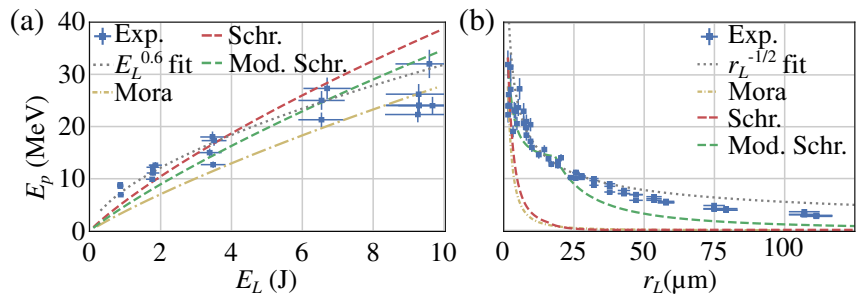

FIG. 4. (a) $E_{p}$ against $E_{L}$ for energy scan and (b) $r_{L}$ for focal scan. Gray dots represent $E_{L}^{0.6}$ and $r_{L}^{-1 / 2}$ power law fits, yellow, red, and green dashes are the Mora, Schreiber, and modified models, respectively. Error bars are from uncertainty in laser parameters and TOF accuracy.
These observations were compared to widely used sheath models. The Mora model [36] was calculated following the assumptions in Ref. [38] except for $T_{e}$ and $\theta_{e}$ which were taken from experimental data (Fig. 2) and using conversion efficiencies of laser energy into electrons $\eta=0.07$ as a free variable to fit maximum proton energy at best focus (Fig. 4). This $\eta$ is significantly lower than expected for such high intensities, and the validity of an isothermal approximation for $\sim$ fs lasers is doubtful [78]. We also plot the Schreiber model, which assumes a quasistatic potential generated by a finite size surface charge of radius $R=r+d \tan \theta$ on the target surface [42], where $r=r_{L} / \sqrt{2 \ln 2}$ is the focus radius $\left(1 / e^{2}\right)$ and $\theta=\theta_{e} / 2 \sqrt{\ln 2}$ is the electrons half-angle $(1 / e)$. The predictions of the model are shown with the red dashed line in Figs. 4(a), 4(b), using $\tau_{L}=40 \mathrm{fs}, d=5 \mu \mathrm{m}$, and $\theta_{E}$ from the linear fits in Fig. 2(d). The laser energy input to the model is $E_{L}^{\prime}=S E_{L}$ where $S$ is the experimental Strehl ratio $S \approx 0.5$ [46] to include the difference in fluence between the experimental and diffraction limited spot, i.e., not all laser energy is usefully focused. We apply the widely used scaling $\eta=1.2 \times 10^{-15} I_{L}^{3 / 4}\left(\mathrm{~W} \mathrm{~cm}^{-2}\right)$ up to a maximum $\eta=0.5[38,42,79,80]$, using the experimentally inferred $I_{L}$ (the discontinuity at $\eta=0.5$ also causes a discontinuity in the model). Despite these assumptions, the model reasonably reproduces the $E_{L}$ scan in Fig. 4(a) without free variables, demonstrating its appropriateness for tightly focused spots. However, both models severely underestimate $E_{p}$ for larger focal spots [Fig. 4(b)].

This model assumes electrons only contribute once to the sheath, accelerating ions during a time $\tau=\tau_{L}$ over which the equation of motion is integrated [42]. However, electrons recirculating between the front and rear surfaces can increase the accelerating potential [81,82]. For small sheath size $R$, the recirculating electrons are unlikely to reappear within the same area. However, for larger $R$, recirculated electrons can contribute to the sheath. Considering an average electron velocity perpendicular to the target $v_{e T} \approx c / 2$, they escape after $\tau_{\text {esc }} \approx 2 R / c$, resulting in an acceleration time calculated by adding in quadrature $\tau=\sqrt{\tau_{L}^{2}+\tau_{\text {esc }}^{2}}$ [40]. Furthermore, the sheath size is modified due to the $45^{\circ}$ angle of incidence to $R^{\prime} \approx r+\sqrt{2} d \tan \theta$, reducing the sheath potential for small $r_{L}$. The resulting estimates are plotted in Fig. 4 (red dashes), resulting in agreement over 3 orders of magnitude in intensity. This energy enhancement for large spots leads to weak $E_{p}$ scaling with reducing $r_{L}$. We note that for current fs-class lasers, timescales are too short for magnetic fields to suppress ion energies [83]. Although our demonstration of proton beams up to $30 \mathrm{MeV}$ at $0.1 \mathrm{~Hz}$ is promising for midenergy applications, generation of higher energies for applications such as radiotherapy using sheath acceleration requires increased laser energy, motivating research into alternative acceleration schemes. 
To conclude, due to insufficient acceleration length at ultrahigh intensities we observed a focal-spot dependent saturation of electron temperature. The resultant sheath accelerated protons showed weak energy scaling when increasing intensity by tightly focusing due to a decrease in effective acceleration time. Therefore, we have demonstrated fundamental limitations of using very tightly focused spots in ultraintense laser-solid interactions. Deviations from scaling laws are important for applications using lasers operating at the high-intensity frontier.

We are grateful to the J-KAREN-P operations team for their support. This work was supported by a JSPS Postdoctoral Fellowship and Kakenhi Project No. 15F15772, JST-MIRAI R\&D Program No. JPMJ17A1 and partially supported by JST PRESTO Grant No. JPMJPR16P9 and Kakenhi 10K05506. The EPOCH code was in part funded by the UK EPSRC Grants No. EP/G054950/1, No. EP/G056803/1, No. EP/ G055165/1, and No. EP/M022463/1. FLASH was developed by the DOE NNSA ASC and NSF-supported FCCS at the University of Chicago.

*dover.nicholas@qst.go.jp

[1] H. Daido, M. Nishiuchi, and A. S. Pirozhkov, Rep. Prog. Phys. 75, 056401 (2012).

[2] A. Macchi, M. Borghesi, and M. Passoni, Rev. Mod. Phys. 85, 751 (2013).

[3] C. M. Brenner, S. R. Mirfayzi, D. R. Rusby, C. Armstrong, A. Alejo, L. A. Wilson, R. Clarke, H. Ahmed, N. M. H. Butler, D. Haddock et al., Plasma Phys. Controlled Fusion 58, 014039 (2016).

[4] M. Tabak, J. Hammer, M. E. Glinsky, W. L. Kruer, S. C. Wilks, J. Woodworth, E. M. Campbell, M. D. Perry, and R. J. Mason, Phys. Plasmas 1, 1626 (1994).

[5] R. Kodama, P. A. Norreys, K. Mima, A. E. Dangor, R. G. Evans, H. Fujita, Y. Kitagawa, K. Krushelnick, T. Miyakoshi, N. Miyanaga et al., Nature (London) 412, 798 (2001).

[6] M. Roth, T. E. Cowan, M. H. Key, S. P. Hatchett, C. Brown, W. Fountain, J. Johnson, D. M. Pennington, R. A. Snavely, S. C. Wilks et al., Phys. Rev. Lett. 86, 436 (2001).

[7] A. Yogo, K. Sato, M. Nishikino, M. Mori, T. Teshima, H. Numasaki, M. Murakami, Y. Demizu, S. Akagi, S. Nagayama et al., Appl. Phys. Lett. 94, 181502 (2009).

[8] D. Doria, K. F. Kakolee, S. Kar, S. K. Litt, F. Fiorini, H. Ahmed, S. Green, J. C. G. Jeynes, J. Kavanagh, D. Kirby et al., AIP Adv. 2, 011209 (2012).

[9] B. Dromey, M. Coughlan, L. Senje, M. Taylor, S. Kuschel, B. Villagomez-Bernabe, R. Stefanuik, G. Nersisyan, L. Stella, J. Kohanoff et al., Nat. Commun. 7, 10642 (2016).

[10] M. Barberio, M. Scisciò, S. Vallières, F. Cardelli, S. N. Chen, G. Famulari, T. Gangolf, G. Revet, A. Schiavi, M. Senzacqua, and P. Antici, Nat. Commun. 9, 372 (2018).

[11] G. A. Mourou, T. Tajima, and S. V. Bulanov, Rev. Mod. Phys. 78, 309 (2006).
[12] M. Roth, D. Jung, K. Falk, N. Guler, O. Deppert, M. Devlin, A. Favalli, J. Fernandez, D. Gautier, M. Geissel et al., Phys. Rev. Lett. 110, 044802 (2013).

[13] C. Danson, D. Hillier, N. Hopps, and D. Neely, High Power Laser Sci. Eng. 3, e3 (2015).

[14] A. Di Piazza, C. Müller, K. Z. Hatsagortsyan, and C. H. Keitel, Rev. Mod. Phys. 84, 1177 (2012).

[15] S. C. Wilks, W. L. Kruer, M. Tabak, and A. B. Langdon, Phys. Rev. Lett. 69, 1383 (1992).

[16] T. Kluge, T. Cowan, A. Debus, U. Schramm, K. Zeil, and M. Bussmann, Phys. Rev. Lett. 107, 205003 (2011).

[17] J. D. Lindl and P. K. Kaw, Phys. Fluids 14, 371 (1971).

[18] Y. Sentoku, K. Mima, H. Ruhl, Y. Toyama, R. Kodama, and T. E. Cowan, Phys. Plasmas 11, 3083 (2004).

[19] A. Sorokovikova, A. V. Arefiev, C. McGuffey, B. Qiao, A. P. L. Robinson, M. S. Wei, H. S. McLean, and F. N. Beg, Phys. Rev. Lett. 116, 155001 (2016).

[20] M. I. K. Santala, M. Zepf, I. Watts, F. N. Beg, E. Clark, M. Tatarakis, K. Krushelnick, A. E. Dangor, T. McCanny, I. Spencer et al., Phys. Rev. Lett. 84, 1459 (2000).

[21] R. J. Gray, D. C. Carroll, X. H. Yuan, C. M. Brenner, M. Burza, M. Coury, K. L. Lancaster, X. X. Lin, Y. T. Li, D. Neely et al., New J. Phys. 16, 113075 (2014).

[22] M. G. Haines, M. S. Wei, F. N. Beg, and R. B. Stephens, Phys. Rev. Lett. 102, 045008 (2009).

[23] F. N. Beg, A. R. Bell, A. E. Dangor, C. N. Danson, A. P. Fews, M. E. Glinsky, B. A. Hammel, P. Lee, P. A. Norreys, and M. Tatarakis, Phys. Plasmas 4, 447 (1997).

[24] R. A. Snavely, M. H. Key, S. P. Hatchett, T. E. Cowan, M. Roth, T. W. Phillips, M. A. Stoyer, E. A. Henry, T. C. Sangster, M. S. Singh et al., Phys. Rev. Lett. 85, 2945 (2000).

[25] E. L. Clark, K. Krushelnick, M. Zepf, F. N. Beg, M. Tatarakis, A. Machacek, M. I. K. Santala, I. Watts, P. A. Norreys, and A. E. Dangor, Phys. Rev. Lett. 85, 1654 (2000).

[26] M. Nishiuchi, H. Sakaki, T. Zh. Esirkepov, K. Nishio, T. A. Pikuz, A. Ya. Faenov, I. Y. Skobelev, R. Orlandi, H. Sako, A. S. Pirozhkov et al., Phys. Plasmas 22, 033107 (2015).

[27] J. Schreiber, P. R. Bolton, and K. Parodi, Rev. Sci. Instrum. 87, 071101 (2016).

[28] T. Esirkepov, M. Borghesi, S. V. Bulanov, G. Mourou, and T. Tajima, Phys. Rev. Lett. 92, 175003 (2004).

[29] A. P. L. Robinson, M. Zepf, S. Kar, R. G. Evans, and C. Bellei, New. J. Phys. 10, 013021 (2008).

[30] J. Denavit, Phys. Rev. Lett. 69, 3052 (1992).

[31] L. O. Silva, M. Marti, J. R. Davies, R. A. Fonseca, C. Ren, F. S. Tsung, and W. B. Mori, Phys. Rev. Lett. 92, 015002 (2004).

[32] F. Fiuza, A. Stockem, E. Boella, R. A. Fonseca, L. O. Silva, D. Haberberger, S. Tochitsky, C. Gong, W. B. Mori, and C. Joshi, Phys. Rev. Lett. 109, 215001 (2012).

[33] L. Yin, B. J. Albright, B. M. Hegelich, and J. C. Fernández, Laser Part. Beams 24, 291 (2006).

[34] A. Henig, D. Kiefer, K. Markey, D. C. Gautier, K. A. Flippo, S. Letzring, R. P. Johnson, T. Shimada, L. Yin, B. J. Albright et al., Phys. Rev. Lett. 103, 045002 (2009).

[35] S. Palaniyappan, C. Huang, D. C. Gautier, C. E. Hamilton, M. A. Santiago, C. Kreuzer, A. B. Sefkow, R. C. Shah, and J. C. Fernández, Nat. Commun. 6, 10170 (2015).

[36] P. Mora, Phys. Rev. Lett. 90, 185002 (2003).

[37] P. Mora, Phys. Rev. E 72, 056401 (2005). 
[38] J. Fuchs, P. Antici, E. D'Humières, E. Lefebvre, M. Borghesi, E. Brambrink, C. A. Cecchetti, M. Kaluza, V. Malka, M. Manclossi et al., Nat. Phys. 2, 48 (2006).

[39] M. Passoni, L. Bertagna, and A. Zani, New J. Phys. 12, 045012 (2010).

[40] C. M. Brenner, P. McKenna, and D. Neely, Plasma Phys. Controlled Fusion 56, 084003 (2014).

[41] L. Robson, P. T. Simpson, R. J. Clarke, K. W. D. Ledingham, F. Lindau, O. Lundh, T. McCanny, P. Mora, D. Neely, C.-G. Wahlström et al., Nat. Phys. 3, 58 (2007).

[42] J. Schreiber, F. Bell, F. Grüner, U. Schramm, M. Geissler, M. Schnürer, S. Ter-Avetisyan, B. M. Hegelich, J. Cobble, E. Brambrink et al., Phys. Rev. Lett. 97, 045005 (2006).

[43] K. Zeil, S. D. Kraft, S. Bock, M. Bussmann, T. E. Cowan, T. Kluge, J. Metzkes, T. Richter, R. Sauerbrey, and U. Schramm, New J. Phys. 12, 045015 (2010).

[44] D. T. Offermann, K. A. Flippo, J. Cobble, M. J. Schmitt, S. A. Gaillard, T. Bartal, D. V. Rose, D. R. Welch, M. Geissel, and M. Schollmeier, Phys. Plasmas 18, 056713 (2011).

[45] B. Aurand, L. Senje, K. Svensson, M. Hansson, A. Higginson, A. Gonoskov, M. Marklund, A. Persson, O. Lundh, D. Neely et al., Phys. Plasmas 23, 023113 (2016).

[46] A. S. Pirozhkov, Y. Fukuda, M. Nishiuchi, H. Kiriyama, A. Sagisaka, K. Ogura, M. Mori, M. Kishimoto, H. Sakaki, N. P. Dover et al., Opt. Express 25, 20486 (2017).

[47] H. Kiriyama, A. S. Pirozhkov, M. Nishiuchi, Y. Fukuda, K. Ogura, A. Sagisaka, Y. Miyasaka, M. Mori, H. Sakaki, N. P. Dover et al., Opt. Lett. 43, 2595 (2018).

[48] M. Nishiuchi, H. Kiriyama, H. Sakaki, N. P. Dover, K. Kondo, A. S. Pirozhkov, A. Sagisaka, Y. Fukuda, K. Nishitani, T. Miyahara et al., Proc. SPIE Int. Soc. Opt. Eng. 10241 (2017).

[49] See Supplemental Material at http://link.aps.org/ supplemental/10.1103/PhysRevLett.124.084802 for more details of the laser and diagnostic setup, which includes Refs. [46,50-60] and for more description of the FLASH simulation setup, which includes Refs. [61-63].

[50] H. Sakaki et al. (to be published).

[51] L. Gremillet, G. Bonnaud, and F. Amiranoff, Phys. Plasmas 9, 941 (2002).

[52] M. S. Wei, F. N. Beg, E. L. Clark, A. E. Dangor, R. G. Evans, A. Gopal, K. W. D. Ledingham, P. McKenna, P. A. Norreys, M. Tatarakis et al., Phys. Rev. E 70, 056412 (2004).

[53] M. Manclossi, J. J. Santos, D. Batani, J. Faure, A. Debayle, V. T. Tikhonchuk, and V. Malka, Phys. Rev. Lett. 96, 125002 (2006).

[54] C. M. Huntington, A. G. R. Thomas, T. Matsuoka, V. Chvykov, G. Kalintchenko, S. Kneip, Z. Najmudin, C. Palmer, V. Yanovsky, A. Maksimchuk et al., Phys. Rev. Lett. 106, 105001 (2011).

[55] J. Peebles, A. V. Arefiev, S. Zhang, C. McGuffey, M. Spinks, J. Gordon, E. W. Gaul, G. Dyer, M. Martinez, M. E. Donovan et al., Phys. Rev. E 98, 053202 (2018).

[56] S. Nakamura, Y. Iwashita, A. Noda, T. Shirai, H. Tongu, A. Fukumi, M. Kado, A. Yogo, M. Mori, and S. Orimo, Jpn. J. Appl. Phys. 45, L913 (2006).

[57] K. Nemoto, A. Maksimchuk, S. Banerjee, K. Flippo, G. Mourou, D. Umstadter, and V. Yu. Bychenkov, Appl. Phys. Lett. 78, 595 (2001).
[58] J. Fuchs, Y. Sentoku, S. Karsch, J. Cobble, P. Audebert, A. Kemp, A. Nikroo, P. Antici, E. Brambrink, A. Blazevic et al., Phys. Rev. Lett. 94, 045004 (2005).

[59] N. P. Dover, M. Nishiuchi, H. Sakaki, M. A. Alkhimova, A. Ya. Faenov, Y. Fukuda, H. Kiriyama, A. Kon, K. Kondo, K. Nishitani et al., Rev. Sci. Instrum. 88, 073304 (2017).

[60] M. Schollmeier, M. Geissel, A. B. Sefkov, and K. A. Flippo, Rev. Sci. Instrum. 85, 043305 (2014).

[61] S. Faik, A. Tauschwitz, and I. Iosilevskiy, Comput. Phys. Commun. 227, 117 (2018).

[62] R. M. More, K. H. Warren, D. A. Young, and G. B. Zimmerman, Phys. Fluids 31, 3059 (1988).

[63] A. J. Kemp and J. Meyer-ter-Vehn, Nucl. Instrum. Methods Phys. Res., Sect. A 415, 674 (1998).

[64] J. J. Santos, F. Amiranoff, S. D. Baton, L. Gremillet, M. Koenig, E. Martinolli, M. Rabec Le Gloahec, C. Rousseaux, D. Batani, A. Bernardinello et al., Phys. Rev. Lett. 89, 025001 (2002).

[65] J. Fuchs, T. E. Cowan, P. Audebert, H. Ruhl, L. Gremillet, A. Kemp, M. Allen, A. Blazevic, J.-C. Gauthier, M. Geissel et al., Phys. Rev. Lett. 91, 255002 (2003).

[66] M. Coury, D. C. Carroll, A. P. L. Robinson, X. H. Yuan, C. M. Brenner, M. Burza, R. J. Gray, M. N. Quinn, K. L. Lancaster, Y. T. Li et al., Appl. Phys. Lett. 100, 074105 (2012).

[67] J. S. Green, V. M. Ovchinnikov, R. G. Evans, K. U. Akli, H. Azechi, F. N. Beg, C. Bellei, R. R. Freeman, H. Habara, R. Heathcote et al., Phys. Rev. Lett. 100, 015003 (2008).

[68] L. Willingale, A. V. Arefiev, G. J. Williams, H. Chen, F. Dollar, A. U. Hazi, A. Maksimchuk, M. J.-E. Marley, W. Nazarov, T. Z. Zhao, and C. Zulick, New J. Phys. 20, 093024 (2018).

[69] Z. Gong, A. P. L. Robinson, X. Q. Yan, and A. V. Arefiev, Plasma Phys. Controlled Fusion 61, 035012 (2019).

[70] Z. Gong, F. Mackenroth, T. Wang, X. Q. Yan, T. Toncian, and A. V. Arefiev, arXiv:1811.00425.

[71] A. V. Arefiev, V. N. Khudik, A. P. L. Robinson, G. Schvets, L. Willingale, and M. Schollmeier, Phys. Plasmas 23, 056704 (2016).

[72] V. Khudik, A. Arefiev, X. Zhang, and G. Schvets, Phys. Plasmas 23, 103108 (2016).

[73] T. D. Arber, K. Bennett, C. S. Brady, A. Lawrence-Douglas, M. G. Ramsay, N. J. Sircombe, P. Gillies, R. G. Evans, H. Schmitz, A. R. Bell, and C. P. Ridgers, Plasma Phys. Controlled Fusion 57, 113001 (2015).

[74] D. J. Stark, T. Toncian, and A. V. Arefiev, Phys. Rev. Lett. 116, 185003 (2016).

[75] T. Kluge, S. A. Gaillard, K. A. Flippo, T. Burris-Mog, W. Enghardt, B. Gall, M. Geissel, A. Helm, S. D. Kraft, T. Lockard et al., New J. Phys. 14, 023038 (2012).

[76] H. Habara, K. Adumi, T. Yabuuchi, T. Nakamura, Z. L. Chen, M. Kashihara, R. Kodama, K. Kondo, G. R. Kumar, L. A. Lei et al., Phys. Rev. Lett. 97, 095004 (2006).

[77] L. Chopineau, A. Leblanc, G. Blaclard, A. Denoeud, M. Thévenet, J.-L. Vay, G. Bonnaud, Ph. Martin, H. Vincenti, and F. Quéré, Phys. Rev. X 9, 011050 (2019).

[78] K. Zeil, J. Metzkes, T. Kluge, M. Bussmann, T. E. Cowan, S. D. Kraft, R. Sauerbrey, and U. Schramm, Nat. Commun. 3, 874 (2012). 
[79] M. H. Key, M. D. Cable, T. E. Cowan, K. G. Estabrook, B. A. Hammel, S. P. Hatchett, E. A. Henry, D. E. Hinkel, J. D. Kilkenny, J. A. Koch et al., Phys. Plasmas 5, 1966 (1988).

[80] J. Yu, Z. Jiang, J. C. Kieffer, and A. Krol, Phys. Plasmas 6, 1318 (1999).

[81] A. J. Mackinnon, Y. Sentoku, P. K. Patel, D. W. Price, S. Hatchett, M. H. Key, C. Andersen, R. Snavely, and R. R. Freeman, Phys. Rev. Lett. 88, 215006 (2002).
[82] S. Buffechoux, J. Psikal, M. Nakatsutsumi, L. Romagnani, A. Andreev, K. Zeil, M. Amin, P. Antici, T. Burris-Mog, A. Compant-La-Fontaine et al., Phys. Rev. Lett. 105, 015005 (2010).

[83] M. Nakatsutsumi, Y. Sentoku, A. Korzhimanov, S. N. Chen, S. Buffechoux, A. Kon, B. Atherton, P. Audebert, M. Geissel, L. Hurd et al., Nat. Commun. 9, 280 (2018). 